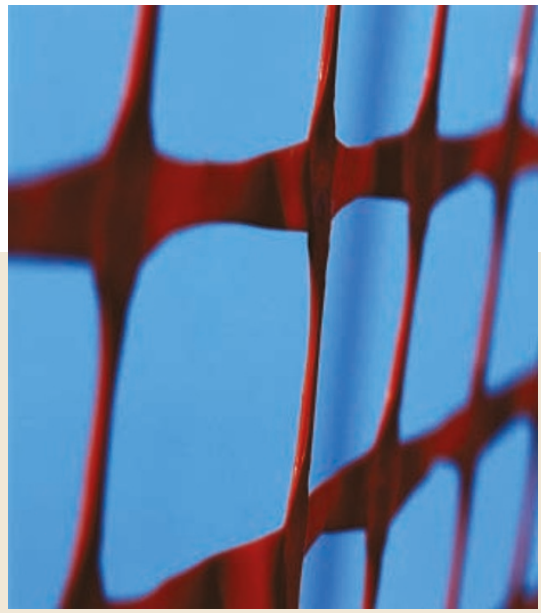

gigh the jury is still out on wheth insoluble deposits that are a characteristic feature of so many neurodegenerative diseases are causative or simply symptomatic, the search to find inhibitors of protein aggregation in such diseases seems to be one of the few possible routes to muchneeded therapies. In a paper published in the Proceedings of the National Academy of Sciences, Erich Wanker and colleagues have stepped up the pace of the search by introducing a simple method for highthroughput screening of compounds that block the self-assembly of poly-glutaminecontaining huntingtin aggregates, the

pathological hallmark of Huntington's disease (HD).

$\mathrm{HD}$, and the formation of aggregates, results when a threshold number of glutamine residues ( $>37)$, which are encoded in exon 1 of the huntingtin gene, are present in the huntingtin protein. The screening process that the authors have developed relies on the fact that aggregated huntingtin is retained by cellulose-acetate membranes, whereas unaggregated huntingtin is not. After incubating a polyglutamine-containing HD exon 1 fusion protein with potential inhibitor compounds in a 384-well format, the reaction mixtures were filtered through cellulose acetate and captured aggregates were revealed by immunoblotting. Testing a Merck compound library of $\sim 184,000$ molecules exposed five groups of structurally similar compounds that reduced the level of aggregation.

The authors reveal the identity of one of the compound groups, which are benzothiazoles that are closely related to Riluzole, a compound that is already used for the treatment of amyotrophic lateral sclerosis. The most potent in vitro inhibitors were, however, shown to be toxic in a cell-based assay of aggregation inhibition, highlighting the difficulty in translating in vitro screening results into therapy.

(9) References and links ORIGINAL RESEARCH PAPER Heiser, V. et al. Identification of benzothiazoles as potential polyglutamine aggregation inhibitors of Huntington's disease by using an automated filter retardation assay. Proc. Natt Acad. Sci. USA 2002 Aug 28 (doi: 10.1073/pnas. 182426599)

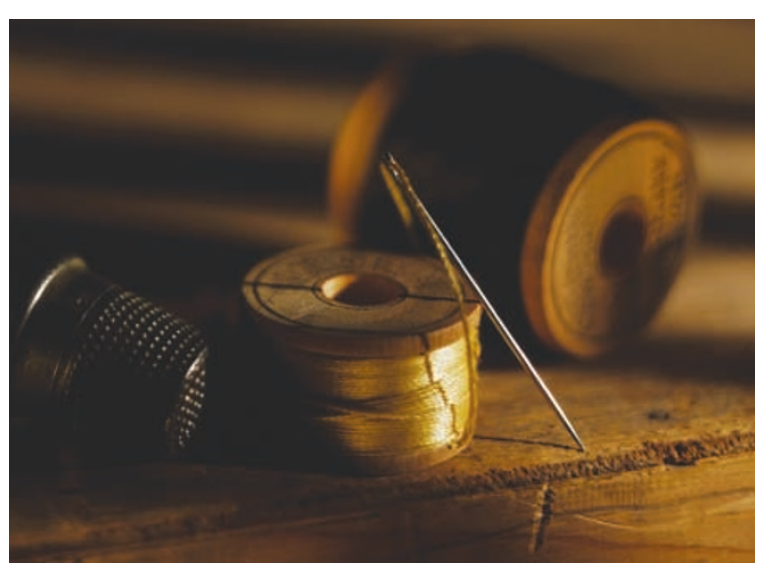

Natural products and their derivatives have traditionally been the most common source of drugs, and still represent more than $30 \%$ of the current pharmaceutical market. However, in much of the pharmaceutical industry, natural products have fallen out of favour in screening programmes, in part owing to the challenges of synthesizing derivatives of what are generally highly complex structures. One possible approach to this problem is to harness the natural enzymes that synthesize such compounds to produce novel derivatives. Writing in Nature, Walsh and colleagues describe the use of an isolated bacterial enzyme in combination with conventional solid-phase peptide synthesis to produce hundreds of compounds related to the natural antibiotic tyrocidine $\mathrm{A}$, several of which have enhanced therapeutic utility.

Tyrocidine A is a cyclic decapeptide, and like many pharmacologically active peptidic natural products is synthesized non-ribosomally by

\title{
ANTIBACTERIAL DRUGS
}

\section{Stitching together naturally}

several large, multidomain enzymes known as peptide synthetases. Such enzymes can be thought of as molecular assembly lines made up of 'modules', each of which incorporates one amino acid into the growing peptide chain before passing it on to the next module. The peptide chain is attached to the enzymes by a thioester tether throughout the synthesis, and once the chain is complete, it is released from the final module by a thioesterase domain. In the case of tyrocidine $\mathrm{A}$, this domain also catalyses specific peptide cyclization, a reaction that is synthetically highly challenging, thus inhibiting investigation of the activity of related cyclic decapeptides.

Building on the close analogy between enzymatic and solid-phase peptide synthesis, and previous work that had shown that the isolated thioesterase domain could catalyse the cyclization of a range of peptide substrates, the authors synthesized more than 300 linear decapeptides using solid-phase synthesis, and assessed whether they could be cyclized using the thioesterase domain. Many were successfully cyclized, and important insights into the sequence requirements of the domain were obtained from comparisons of the ratio of cyclized to non-cyclized products. Tests of the novel products against the bacteria Bacillus subtilis were then used to clarify the influence of the nature of the component amino acids on antibiotic activity.
Tyrocidine A - which is thought to act by inserting into cell membranes and thereby lysing the cells - has limited clinical utility, owing to poor selectivity for bacterial cell membranes over eukaryotic cell membranes. Using the knowledge gained from the structure-activity studies, the authors synthesized $\sim 200$ decapeptides, and tested them simultaneously in antibacterial assays using $B$. subtilis and for their ability to lyse human blood cells. Many of the analogues had an improved therapeutic profile compared with tyrocidine $\mathrm{A}$, and two of the best analogues, which were synthesized on a preparative scale, also had an improved therapeutic profile against a broad spectrum of Gram-positive and Gramnegative bacteria. These compounds are promising candidates for further evaluation, and establish the potential of thioesterase domains in the combinatorial biomimetic synthesis of novel molecules. More generally, as understanding of the biosynthesis of natural products continues to grow, many diverse libraries of natural-product derivatives with potentially valuable activities could become accessible by such approaches.

(Q) References and links Peter Kirkpatrick ORIGINAL RESEARCH PAPER Kohil, R. M. et al. Biomimetic synthesis and optimization of cyclic peptide antibiotics. Nature 418, 658-661 (2002)

FURTHER READING Cane, D. E. et al. Harnessing the biosynthetic code: combinations, permutations and mutations. Science 282, 63-68 (1998) 